\title{
Metástases cranianas Considerações sobre 21 casos
}

\author{
Carlos Umberto Pereira', Mirela Secundo Melo². \\ Departamento de Medicina da Universidade Federal de Sergipe \\ Serviço de Neurocirurgia do Hospital João Alves Filho (Aracaju, SE)
}

\section{RESUMO}

Contexto: Metástases cranianas são raras, podem derivar de todas as neoplasias epiteliais e acometem principalmente os idosos. Objetivo: Analisar pacientes com metástases cranianas, atendidos em nosso serviço. Método: Estudo prospectivo e descritivo. Foram estudados 21 pacientes de ambos os gêneros admitidos no Serviço de Neurocirurgia do Hospital João Alves Filho (Aracaju, SE), durante o período de janeiro de 1999 a março de 2009, com o diagnóstico de metástase craniana, colhendo dados de exame físico, quadro clínico, foco primário, exames de imagens, histopatológicos, tratamento e prognóstico. Resultados: Houve predomínio do sexo feminino (12:9). As idades variaram entre 1 e 74 anos, com uma média de 51,1 anos. A queixa principal foi cefaleia, seguida de tumoração local. A distribuição do tumor primário foi: mama (5), pulmão (3), fígado (2), próstata (2), tireoide (2), mieloma múltiplo (2), neuroblastoma (2), carcinoma epidermoide (1) e desconhecido (2). A localização das lesões foi única em 13 casos e múltipla em 8, com predomínio no osso parietal. Radiografia simples de crânio, tomografia computadorizada (TC) e ressonância magnética (RM) foram relevantes no diagnóstico, mas a confirmação diagnóstica foi por meio da histopatologia. Comprometimento intracraniano esteve presente em nove casos. Biópsia foi realizada em dez casos, exérese completa da lesão em cinco, parcial em três e tratamento conservador em 3. Radioterapia complementar foi realizada em 16 casos e quimioterapia em 9. Ocorreram 14 óbitos. Conclusão: Metástases cranianas geralmente acometem pessoas idosas com neoplasia primária conhecida. Seu tratamento tem base no estado geral do paciente, na localização e apresenta um prognóstico péssimo.

\section{PALAVRAS-CHAVE}

Neoplasia craniana. Metástases cranianas.

\section{ABSTRACT}

Skull metastasis. Considerations about 21 cases

Background: Skull metastasis are rare, can originate from all ephitelial neoplasms and they occur predominantly in elderly. Objective: To study patients with cranial metastasis admitted in our Service. Methods: In a prospective and descriptive study we analyzed twenty-one cases of skull metastases, admitted in the Serviço de Neurocirurgia do Hospital João Alves Filho (Aracaju, SE) from January 1999 to March 2009, including clinical findings, physical examination, diagnostic images, primary neoplasm, histopathology, treatment and prognosis. Results: Male:female ratio was 9:12; the median age was 51.1 years, ranging from 1 to 74 years. Most patients presented with headache, followed by local swelling. Primary tumor was located in breast (5), lung (3), liver (2), prostate (2), thyroid (2), multiple myeloma (2), neuroblastoma (2), epidermoid carcinoma (1), and unknown (2). There was a single lesion in 13 cases and multiple lesions in 8, and the involvement of the parietal bone was predominant. Skull X-ray, CT and MRI were relevant to the diagnosis, but the confirmation was provided by histopathology. There was intracranial growth of the tumor in nine cases. Needle biopsy was held in ten cases, total excision of the lesion in five, partial in three and conservative treatment was decided in 3 cases. Adjuvant radiotherapy was done in 16 cases and chemotherapy in 9. Forteen patients died. Conclusion: Skull metastasis tend to occur in elderly patients with diagnosis of cancer. Therapeutic decision depends on general condition of the patient and tumor location, and generally the prognosis is poor.

\section{KEY WORDS}

Cranial tumors. Skull metastases.

1 Professor doutor do Departamento de Medicina da Universidade Federal de Sergipe (UFS), neurocirurgião do Hospital João Alves Filho, Aracaju, Sergipe.

2 Doutoranda de Medicina da UFS, bolsista de Iniciação à Pesquisa Pibic/UFS/CNPq. 


\section{Introdução}

As metástases cranianas (MC) consistem em lesões osteolíticas, expansivas e hipervascularizadas localizadas na calota craniana. ${ }^{3,37,49}$ Elas têm baixa morbidade e o diagnóstico ocorre, geralmente, meses ou anos após a detecção da neoplasia primária. Em alguns casos, o diagnóstico pode preceder ao diagnóstico do foco primário. Não são responsáveis pelo óbito dos pacientes na maioria dos casos, que quase sempre está relacionado com a neoplasia primária. ${ }^{37,49}$

As manifestações clínicas diferem dos tumores intracranianos e de outras lesões de natureza inflamatória, traumática ou congênita, à medida que se apresentam como massas indolores, causam com menor frequência déficits neurológicos e acometem geralmente idosos. ${ }^{49}$ As neoplasias primárias que mais ocasionam MC são aquelas localizadas na mama, no pulmão, na tireoide e na próstata. ${ }^{5,13,27,34,55} \mathrm{O}$ tratamento depende de localização, número de metástases, idade e condições clínicas do paciente e apresenta um prognóstico reservado.

Os autores apresentam sua experiência em 21 casos de MC, discutindo o quadro clinico, os exames de imagens, a conduta e o prognóstico.

\section{Revisão da literatura}

As neoplasias cranianas constituem de $1 \%$ a $2 \%$ de todos os tumores ósseos, sendo que as lesões metastáticas são, em geral, muito mais frequentes que os tumores ósseos primários. ${ }^{4}$ As MCs são mais raras que aquelas localizadas em outras partes do esqueleto, e sua frequência é muito menor do que as metástases cerebrais. ${ }^{4,6}$ Tofe e col. ${ }^{51}$ demonstraram que as MCs apresentam uma incidência de $23 \%$ em pacientes com neoplasia primária de mama, pulmão ou próstata, quando submetidos a exame de cintilografia óssea. Nunan e col. ${ }^{43}$ relataram que o crânio pode ser sede de metástases em 11,6\% dos pacientes portadores de câncer. As MCs predominam em pacientes após a quinta década de vida e são geralmente assintomáticas, sendo descobertas principalmente em exames radiológicos de rotina de pacientes portadores de neoplasia primária., ${ }^{3,49}$

As metástases na calota craniana são mais frequentes que aquelas localizadas na base do crânio e na órbita. As metástases localizadas na base do crânio são geralmente de carcinoma de mama, pulmão e próstata. ${ }^{19,32}$

Geralmente, apresentam-se em localizações múltiplas e de aspecto osteolítico, ${ }^{55}$ sendo rara a forma solitária. ${ }^{6}$ Quase todas as neoplasias de origem epitelial são capazes de desenvolver uma $\mathrm{MC}$, porém são mais frequentes nos casos de carcinoma de mama, pulmão, próstata e tireoide. . $^{1,57-9,16,27,28,33,35-37,48,49,51,55}$ Focos primários menos frequentes são carcinomas de bexiga, ${ }^{8}$ renal, ${ }^{13,17,37}$ seminoma testicular, ${ }^{13,37}$ colorretal, ${ }^{13,49}$ melanoma, ${ }^{37,49}$ hepática, ${ }^{12,13,23,27,29,31,38,39,45,56}$ carcinoma cervical ${ }^{37}$ e linfomas. ${ }^{8}$ Os sarcomas como leiomiossarcoma ${ }^{25,30,49}$, osteossarcoma ${ }^{37}$ e sarcoma de Ewing $22,37,49$ também podem desenvolver MC e, nas crianças, o neuroblastoma. ${ }^{11,15,20,35,44,46,55}$ Por causa da conhecida inexistência de vias linfáticas no tecido ósseo, estas alcançam a calota craniana por meio de disseminação hematogênica. . $^{4,714,32,35,49}$

Manifestações clínicas de MC antes da neoplasia primária são raras. ${ }^{31,41,53} \mathrm{~A}$ maioria das MCs é assintomática e achada em autópsias. ${ }^{49} \mathrm{~A}$ literatura médica tem sido pobre na descrição das manifestações clínicas de MC. . $^{13,37,40,54}$ As metástases para base do crânio são ainda mais raras, assim como as descrições de seu quadro clinico. ${ }^{35,49}$ Quando localizada na calota craniana, o sinal mais comum é uma tumoração formada a partir da erosão da tábua óssea externa do crânio. Frequentemente ocorre envolvimento da díploe em toda a sua espessura. Ao expandir através da tábua interna do crânio e comprimir o periósteo, dura-máter e o parênquima cerebral adjacente, essas lesões geralmente se tornam dolorosas. ${ }^{6}$ Ocasionalmente, uma massa metastática localizada no calvário pode comprimir os seios venosos durais, o que pode obstruir o fluxo venoso local ou comprimir nervos cranianos, quando localizada na base do crânio, ${ }^{32,47,49}$ causando uma elevação da pressão intracraniana, com quadro de cefaleia, vômitos e papiledema. ${ }^{13,37,52}$ Contudo, esse efeito só é considerável em casos de metástases de crescimento rápido, em que o surgimento de um fluxo venoso compensatório por veias corticais é ineficaz. ${ }^{13}$ Em alguns casos, déficits neurológicos focais podem resultar de crescimento interno progressivo de uma lesão metastática para o calvário. Outros sintomas neurológicos podem estar presentes como exoftalmia, crises convulsivas e hemiparesia. Podem estar associados a hematomas subdural ou epidural. ${ }^{23,29,41,42,48}$ Em geral, as MCs não são responsáveis pelo óbito, que quase sempre está relacionado ao desenvolvimento da neoplasia primária. ${ }^{37,49}$

Essas lesões são quase exclusivamente de caráter osteolítico, com exceção dos tumores de mama e próstata, que frequentemente se apresentam como lesão osteoblástica. ${ }^{3,6}$ Histologicamente, ocorre uma substituição difusa ou completa do tecido ósseo esponjoso por células neoplásicas como se observa com frequência em processos líticos. ${ }^{6}$

A radiografia simples de crânio demonstra lesões osteolíticas como um defeito ósseo de bordas irregulares, acompanhado de atrofia do osso adjacente, dependendo da velocidade de crescimento da lesão, e são obser- 
vadas em casos de carcinoma de pulmão, útero, trato gastointestinal, tireoide e melanoma. ${ }^{3,644,50}$ As lesões osteoblásticas aparecem como uma área hiperdensa, às vezes com pequenos sítios internos de densidade elevada (flocos de neve) ${ }^{6}$ observadas principalmente em casos de carcinoma de próstata, mama, bexiga e hipernefroma. As MCs de carcinoma de próstata têm geralmente aspecto esclerótico ou misto. Metástases de carcinoma renal e de tireoide têm geralmente aspecto esclerótico e são solitárias. ${ }^{55}$

A tomografia computadorizada (TC) mostra o comprometimento ósseo, detecta melhor a erosão na base do crânio e o grau de invasão parenquimatosa, sendo esta última característica mais bem observada no exame de ressonância magnética (RM), que também é capaz de determinar se há compressão de seio venoso intracraniano e seu grau de comprometimento, além de possibilitar a detecção das lesões em estágios mais precoces. $2,6,22,35,49$

A RM mostra, em T1, áreas focais hipodensas, que são facilmente distinguíveis da hiperdensidade do sinal da medula óssea normal. ${ }^{2}$ Em imagens em T2 com contraste são vistas áreas hiperdensas substituindo o espaço diploico normal e o osso cortical. ${ }^{2}$ A TC e a RM demonstram lesão focal osteolítica ou osteoblástica acometendo ambas tábuas ósseas cranianas e, quando ocorre extensão intracraniana, demonstra uma lesão de forma biconvexa (lenticular) associada com desvio do parênquima cerebral da tábua interna do crânio. ${ }^{35}$

A cintilografia óssea tem sido indicada em casos de MC e com resultados excelentes. . $^{90,35}$

A angiografia cerebral pode ser utilizada para determinação do suprimento do tumor a fim de planejar uma possível embolização vascular prévia à abordagem cirúrgica. ${ }^{6,22}$ Assim, na presença de tumor primário desconhecido, associado a metástases cerebrais e múltiplas lesões osteolíticas no calvário, exames de imagens podem ajudar a definir a natureza maligna da lesão, mas o diagnóstico definitivo é feito por meio do exame histopatológico. ${ }^{6,24}$

Não existem sinais patognomônicos que ajudem no diagnóstico diferencial com outras lesões. ${ }^{4}$ Focos solitários de MC podem ser muito difíceis de se distinguirem, tanto clínica quanto radiologicamente, de tumores primários da calota ou de lesões de natureza traumática, inflamatória ou congênita. ${ }^{2,13}$ Os primeiros incluem lesões benignas como lipoma, hemangioma, displasia fibrosa, osteoma e meningioma; ou lesões malignas como condrossarcoma, osteossarcoma, mieloma múltiplo, sarcoma de Paget e sarcoma Ewing. ${ }^{2}$ Entre as lesões não tumorais temos: cisto dermoide e epidermoide, histiocitose das células de Langerhans, esclerose idiopática e granuloma eosinofílico. ${ }^{2,6}$ As MCs podem ser diferenciadas à medida que apresentam massas geralmente indolores, ocorrem em pacientes de maior idade e sua história da evolução dos sintomas é mais curta. O meningioma, em particular, apresenta diferença no diagnóstico em relação à $\mathrm{MC}$ única pelo comprometimento cerebral e pela localização próxima ao seio longitudinal superior e pelo fato de não existir uma história prévia de neoplasia primária. ${ }^{4,33}$

$\mathrm{O}$ tratamento das MCs baseia-se na ressecção cirúrgica e no uso complementar de radioterapia, quimioterapia quando não há resposta ao tratamento sistêmico ou quando se trata de lesão única e circunscrita. ${ }^{4}$ Normalmente, a ressecção cirúrgica da MC é um procedimento simples, seguro, paliativo e de baixa morbimortalidade. ${ }^{4}$ Justifica-se em alguns casos o procedimento cirúrgico radical quando o estado geral do paciente permite e quando a expectativa de vida também permite essa alternativa de tratamento. ${ }^{4}$ Chan e col. ${ }^{12}$ relataram que o tratamento de $\mathrm{MC}$ inclui radioterapia, cirurgia e cuidados paliativos, para alívio das dores, reduz o risco de sequelas neurológicas e assim melhora ou mantém a qualidade de vida e seu prolongamento. Wecht e Sawaya ${ }^{54}$ indicam cirurgia em lesão da calota craniana, seja ela benigna ou maligna, para estabelecer o diagnóstico, em casos de lesão solitária e para excisão total de lesão benigna e sintomática.

A localização de uma MC sobre um seio venoso intracraniano tem um caráter distinto em relação às demais localizações, principalmente no tocante à ressecção cirúrgica, em razão da alta vascularização da metástase e da infiltração deste que limitam a ressecção dessa lesão. ${ }^{4}$ Nos casos localizados sobre os seios venosos dural da convexidade pode ocorrer hemorragia extensa, assim como necessidade de ligadura ou reconstrução destes para se obter uma excisão radical da lesão. ${ }^{4}$

Tem-se ressaltado recentemente uma melhoria dos resultados no que concerne à sobrevida e à qualidade de vida dos pacientes quando é tomada a decisão cirúrgica. ${ }^{4}$ Considera-se que a ressecção cirúrgica não influencia na progressão da doença, permite ao paciente que evite dor local e desconforto e que possa diminuir os sintomas neurológicos, quando presentes. Deve ser considerada dentro das seguintes circunstâncias: presença de déficit neurológico, destruição óssea maciça, infiltração dural, massa dolorosa, metástase solitária e para confirmação de diagnóstico. Wecht e Sawaya ${ }^{54}$ consideram como critérios para cirurgia: diagnóstico incerto, lesões benignas com alta probabilidade de remoção total, efeito cosmético, lesões malignas que representam o único foco de doença maligna sistêmica que está em remissão, ou lesões de crescimento rápido com sintomas significativos ou não responsivos à radioterapia e à quimioterapia.

Tratamento cirúrgico ou radioterapia local para MC geralmente não prolongam a vida do paciente em razão 
da evolução da neoplasia primária, mas o diagnóstico definitivo por meio da remoção da lesão ou de biópsia é sempre possível. Radiocirurgia tem sido uma opção terapêutica para o tratamento de metástases localizadas na base do crânio como tratamento primário ou em caso de recidiva após realização de radioterapia. ${ }^{32}$

Em casos de MC em estágios avançados e quando há necessidade de alívio dos sintomas, indica-se tratamento cirúrgico. ${ }^{37}$ Alguns casos selecionados podem responder à quimioterapia e à radioterapia. ${ }^{13,37} \mathrm{~A}$ ressecção cirúrgica de MC solitária e até de fragmentos de dura-máter infiltrada não tem influência sobre o desenvolvimento sistêmico da neoplasia primária. ${ }^{13}$ Seu efeito é paliativo, pois promove descompressão, alívio da cefaleia e diminuição dos déficits neurológicos focais. ${ }^{7,13,37} \mathrm{AMC}$ de neoplasia primária da tireoide é associada a péssimo prognóstico. ${ }^{16,18,26}$

\section{Pacientes e métodos}

O presente trabalho é prospectivo e descritivo. Foram estudados 21 pacientes de ambos os gêneros admitidos no Serviço de Neurocirurgia do Hospital João Alves Filho (Aracaju, SE), durante o período de janeiro de 1999 a março de 2009, com o diagnóstico de metástase craniana.

Foram avaliados por meio de anamnese, exame físico, neurológico, radiografia simples de crânio, tomografia computadorizada, ressonância magnética, tratamento e prognóstico.

\section{Resultados}

Houve predomínio do sexo feminino (12:9). As idades variaram entre 1 e 74 anos, com média de 51,1 anos. A queixa principal foi cefaleia, seguida de tumoração local. Quanto ao foco primário, a distribuição foi: mama (5), pulmão (3), fígado (2), próstata (2), tireoide (2), mieloma múltiplo (2), neuroblastoma (2), carcinoma epidermoide (1) e desconhecido (2). As lesões eram únicas em 13 casos e múltiplas em 8 , com predomínio do osso parietal. A radiografia simples de crânio e a TC foram realizadas em todos os casos, e a RM, em nove. O diagnóstico foi estabelecido por meio de exame histopatológico. Comprometimento intracraniano esteve presente em oito casos. Biópsia sem ressecção foi realizada em dez casos, ressecção completa em cinco, parcial em três e conduta conservadora em três casos. Quimioterapia complementar foi realizada em 14 casos e radioterapia em nove. Até a presente publicação ocorreram 14 óbitos.

\section{Discussão}

As MCs podem ocorrer devidas a qualquer neoplasia primária a distância, porém são mais comuns em casos de neoplasias de mama, pulmão, próstata e tireoide. ${ }^{5,14,24,28,30,39}$ As MCs predominam em adultos, ${ }^{14}$ porém podem ocorrer na infância, principalmente em caso de neuroblastoma. ${ }^{11,15,46} \mathrm{Em}$ nosso estudo, a média das idades foi de 51,1 anos, o que se encontra de acordo com a maioria dos autores. ${ }^{4,27,49}$ Houve predomínio no sexo feminino em nosso estudo, talvez decorrente da maior incidência de neoplasia de mama.

A maioria das MCs é assintomática, ${ }^{37}$ sendo, em alguns casos, achadas incidentalmente em exames de rotina para controle da neoplasia primária quando conhecida ou mesmo achadas em autópsia. ${ }^{49} \mathrm{Em}$ nosso estudo o diagnóstico da MC foi sintomática em 12 casos, e a lesão metastática precedeu a da neoplasia primária em nove. A cefaleia foi um achado comum com 14 casos, seguida de tumoração local em dez casos. Três pacientes apresentavam comprometimento do seio longitudinal superior.

As neoplasias primárias mais frequentes foram mama e pulmão, seguidos de próstata e tireoide, fatos estes observados por outros autores. ${ }^{1,5,7,14,24,27,28,48,51}$ Em dois casos não foi possível identificar a lesão primária.

A radiografia simples de crânio e a TC foram significativas no diagnóstico demonstrando a extensão da destruição óssea, e a RM, na avaliação do comprometimento intracraniano da lesão, o que se encontra de acordo com vários autores. ${ }^{2,6,8,35,37,49}$ Em nossa casuística, as lesões foram solitárias em 13 casos, o que também é observado por outros autores. ${ }^{9,13} \mathrm{O}$ comprometimento intracraniano esteve presente em noves casos, dos quais, seis tinham sinais de hipertensão intracraniana, fato este observado por outros autores. . $^{13,37,49,52} \mathrm{O}$ diagnóstico final foi confirmado pelo exame histopatológico em 18 casos.

Biópsia foi realizada em dez casos, ressecção total em cinco, parcial em três e conduta conservadora em três casos; estes apresentavam lesões múltiplas e lesão primaria conhecida. Até a conclusão desse trabalho, ocorreram 14 óbitos, sendo sua maioria como consequência de complicações sistêmicas da doença de base e não em razão de recidiva da metástase. Houve um óbito intraoperatório devido ao sangramento extenso em um caso de comprometimento do seio longitudinal superior. 


\section{Conclusão}

Concluímos que é necessário um acompanhamento rigoroso de pacientes diagnosticados de neoplasia primária por intermédio de um sistema de saúde que facilite seu atendimento para uma detecção precoce e, assim, aumentem-se as chances de sobrevida e melhore-se o prognóstico desses pacientes.

\section{Referências}

1. Akdemir I, Erol FS, Akpolat N, Ozveren MF, Akfirat M, Yashi S. Skull metastasis from thyroid follicular carcinoma with difficult diagnosis of the primary lesion. Neurol Med Chir (Tokyo). 2005;45:205-8.

2. Amaral L, Chiurciu M, Almeida JR, Ferreira NF, Mendonça R, Lima SS. MR imaging for evaluation of lesions of the cranial vault. Arq Neuropsiquiatr (Sao Paulo). 2003;61:521-32.

3. Arana E, Marti-Bonmati L. CT and MR imaging of focal calvarial lesions. Am J Roentgenol. 1999;172:1683-8.

4. Ariel VH, Sergio VB, Miguel DN, Gretel MB, Hector LR, Fernando CA, Oscalyns NC. Metástasis craneales sobre los senos durales de la convexidad. Informe de nueve casos. Rev Mex Neurocirur. 2006;7:240-5.

5. Arseni C, Constantinescu Al. Cerebral and cranial metastases from breast cancer. Neurochirurgia (Stuttg). 1982;25:95-9.

6. Artico M, De Caro GMF, Salvati M, Carloia S, Rastelli E, Wierzbicki V, Manni M. Solitary metastases to the cranial vault. Report of ten cases. J Neurosurg Sci. 2000;44:33-8.

7. Aydin VM, Cezkinmez M, Kizilkilic O, Kayaselcuk F, Sen $\mathrm{O}$, Altinors $\mathrm{N}$. Unusual case of skull metastasis secondary to pancreatic adenocarcinoma. Pathol Oncol Res. 2005;11:182-3.

8. Bisese JH. MRI of cranial metastasis. Sem Ultras CT MRI. 1992;13:473-83.

9. Boxer DI, Todd CE, Coleman R, Fogelman I. Bone secondaries in breast cancer: The solitary metastasis. J Nucl Med. 1989;30:1318-20.

10. Brillman J, Valeriano J, Adatepe MH. The diagnosis of skull base metastases by radionuclide bone scan. Cancer. 1987;59:1887-91.

11. Caran EM, Lopes LF, Camargo B, Feher AT, Marques E, Bianchi A. Disjunção de suturas cranianas em neuroblastoma. Acta Oncol Bras. 1987;7:33-6.

12. Chan $\mathrm{CH}$, Trost N, McKelvie P, Rophael JA, Murphy MA. Unusual case of skull metastasis from hepatocellular carcinoma. ANZ J Surg. 2004;74:710-3.

13. Constans JP, Donzelli R. Surgical features of cranial metastases. Surg Neurol. 1981;15:35-8.

14. Doran ES, Gebarski SS, Hoff TJ. "Tumors of the skull". In: Youmans JR (Ed.). Neurological surgery. Philadelphia: Saunders, 1996. p.2998-3023.

15. Filho AJG, Piza AMBT, Aranega VLJ. Metástases osteoblásticas em neuroblastoma. Relato de caso. Rev Imagem. 1989;11:143-6.

16. Fujita $\mathrm{T}$, Ogasawara $\mathrm{Y}$, Doihara $\mathrm{H}$. Solitary cranial metastasis of thyroid carcinoma 13 years alter primary surgery: report of a case. Surg Today. 2009;39:44-7.
17. Gaetani P, Di leva A, Colombo P, Tancioni F, Aimar E, Debernardi A, Baena RR. Calvarial metastases as clinical presentation of renal carcinoma: report of two cases and review of the literature. Clin Neurol Neurosurg. 2005;107:329-33.

18. Goolden AW, McLaughlin JE, Valentine AR, Pease C. Solitary cerebral metastases from a papillary carcinoma of the thyroid. Postgrad Med J. 1990;66:127-9.

19. Greenberg HS, Deck MD, Vikram B, Chu FC, Posner JB. Metastasis to the base of the skull: clinical findings in 43 patients. Neurology. 1981;31:530-7.

20. Grover SB. Solitary calvarial metastases: An unusual presentation of thoracic neuroblastoma. Ind $\mathrm{J}$ Cancer. 2003;40:120-2.

21. Hashmi R, Uetani M, Ogawa Y, Aziz A. Clinical significance of a solitary hot spot in the skull. Nucl Med Commun. 1999;20:703-10.

22. Hattori T, Yamakawa H, Nakayama N, Kuroda T, Andoh T, Sakai N, et al. Skull metastasis of Ewing's sarcoma. Three case reports. Neurol Med Chir (Tokyo). 1999;39:46-9.

23. Hayashi K, Matsuo T, Kurihara M, Daikoku M, Kitange G, Shibata S. Skull metastasis of hepatocellular carcinoma associated with acute epidural hematoma: a case report. Surg Neurol. 2000;53:379-82.

24. Healy JF, Marshall WH, Brahme FJ, White F. CT of intracranial metastases with skull and scalp involvement. AJNR. 1981;2:335-8.

25. Hernández FA, Barba M, Lorenzo M, Ramos F, Stefanoni G. Metástasis craneal de leiomiosarcoma mediastinal. Informe de un caso y revision de la literatura. Rev Med Hosp Gen Mex. 2003;66:95-8.

26. Hjiyiannakis P, Jefferies $\mathrm{S}$, Harmer CL. Brain metastases in patients with differentiated thyroid carcinoma. Clin Oncol ( $R$ Coll Radiol). 1996;8:327-30.

27. Hsieh CT, Sun JM, Tsai WC, Chiang YH, Liu MY. Skull metastasis from hepatocellular carcinoma. Acta Neurochir (Wien). 2007;85-90.

28. Inci S, Akbay A, Bertan V, Gedikoglu G, Onol B. Solitary skull metastasis from occult thyroid carcinoma. J Neurosurg Sci. 1994;38:63-6.

29. Kanai R, Kubota H, Terada T, Hata T, Tawaraya E, Fujii K. Spontaneous epidural hematoma due to skull metastasis of hepatocellular carcinoma. J Clin Neurosci. 2009;16: 137-40.

30. Komata T, Takahashi S, Konno K, Iwanaga K, Takahashi H. Metastatic leiomyosarcoma of the skull: case report. Neurol Med Chir (Tokyo). 1994;34:180-2.

31. Kuratsu J, Murakami M, Uemura S, Ushio Y. Brain and skull metastases of hepatic or pancreatic cancer. Report of six cases. Neurol Med Chir (Tokyo). 1990;30:476-82.

32. Laigle-Donadey F, Taillibert S, Martin-Duverneuil N, Hildebrand J, Delattre JY. Skull-base metastases. J Neurooncol. 2005;75:63-9.

33. Lippman SM, Buzaid AC, lacono RP, Steinbronn DV, Stanisic $\mathrm{TH}$, Rennels MA, et al. Cranial metastases from prostate cancer simulating meningioma: report of two cases and review of the literature. Neurosurgery. 1986;19:820-3.

34. Long DM, Kieffer SA, Chou SN. Tumorous lesions of the skull. In: Neurological Surgery. Youmans JR, editor. Philadelphia: WB Saunders, 1973. p.1231-74.

35. Maroldi R, Ambrosi C, Farina D. Metastatic disease of the brain: extra-axial metastases (skull, dura, leptomeningeal) and tumor spread. Eur Radiol. 2005;15:617-26.

36. Mendes VC, Linhares P, Fonseca E, Ribeiro-Silva ML. Giant skull metastasis of Hürthle cell variant of thyroid's papillary carcinoma. J Neurooncol. 2008;6:311-2. 
37. Michael CB, Gokaslan ZL, Demonte F, McCutcheon IE, Sawaya R, Lang FF. Surgical resection of calvarial metastases overlying dural sinuses. Neurosurgery. 2001;48: 745-55.

38. Miura T, Hirabuki N, Kozuka T. Case report: cranial metastasis from hepatocellular carcinoma. Clin Radiol. 1990; 42:445-6.

39. Murakami R, Korogi Y, Sakamoto Y, Takahashi M, Okuda T, Yasunaga T, et al. Skull metastasis from hepatocellular carcinoma: CT, MR and angiographic findings. Acta Radiol. 1995;36:597-602

40. Nagamine Y, Suzuki J, Katakura R, Yoshimoto T, Matoba $\mathrm{N}$, Takaya K. Skull metastasis of thyroid carcinoma. Study of 12 cases. J Neurosurg. 1985;63:526-31.

41. Nakagawa $\mathrm{Y}$, Yoshino E, Suzuki K, Tatebe A, Adachi H. Spontaneous epidural hematoma from a hepatocellular carcinoma metastasis to the skull. Case report. Neurol Med Chir (Tokyo). 1992;32:300-2.

42. Nakao S, Sato S, Fukumitsu T, Ogata M, Shirane H. Cranial metastasis of hepatocellular carcinoma. Report of three cases. Neurol Med Chir (Tokyo). 1985;25:229-34.

43. Nunan TO, Clarke SEM, Coakley AJ, Wells CP, Halls FM, Entwhistle $P$. Is the skull view necessary in routine gamma camera bone scanning? Nucl Med Commun. 1985;6:37-40.

44. Pascual-Castroviejo I, Lopez-Martin V, Rodriguez-Costa T, Pascual-Pascual JI. Radiological and anatomical aspects of the cranial metastases of neuroblastomas. Neuroradiology. 1975;9:33-8.

45. Pereira CU, Santos VBO, Ayala MAR, Gonzaga AKV, Santos TBO. Cerebral metastases as the primary manifestations of a hepatocellular carcinoma. Rev Chil Neurocirug. 1999;13:28-31.

46. Pereira CU, Morais DCR, Tavares GA, Dantas MC. Neuroblastoma metastático simulando hematoma subdural. Relato de caso. J Bras Neurocir. 2003;14:25-8.

47. Ranson DT, Dinapoli RP, Richardson RL. Cranial nerve lesions due to base of the skull metastases in prostate carcinoma. Cancer. 1990;65:586-9.
48. Simmons NE, Elias WJ, Henson SL, Laws ER. Small cell lung carcinoma causing epidural hematoma: case report. Surg Neurol. 1999;51:56-9.

49. Stark AM, Eichmann T, Mehdorn HM. Skull metastases: clinical features, differential diagnosis, and review of the literature. Surg Neurol.2003;60:219-26.

50. Taveras JM, Wood EH. Diagnostic neuroradiology. Baltimore: Williams \& Wilkins Co., 1964.

51. Tofe AJ, Francis MD, Harvey WJ. Correlation of neoplasms with incidence and localization of skeletal metastases: an analysis of 1.355 diphosphonate bone scans. J Nucl Med. 1975;16:986-89.

52. Varela-Hernández A, Cerrón-Rojas VR, Herrera-Lorenzo O, Infante J, Garcia-Calzada J, Casares F, Morciego S. Hipertensión endocraneal. Rev Neurol. 2002;34:1152-61.

53. Wakisaka S, Tashiro M, Nakao S, Kita T, Kisanuki H, Kinoshita K. Intracranial and orbital metastasis of hepatocellular carcinoma. Reporto f two cases. Neurosurgery. 1990;26: 863-6.

54. Wecht DA, Sawaya R. Lesions of the calvarial: surgical experience with 42 patients. Ann Surg Oncol. 1997;4:28-36.

55. Yalçin Ö, Yildirim T, Kizilkiliç O, Hürcan CE, Koç Z, Aydin V, et al. CT and MRI findings in calvarial non-infectious lesions. Diagn Interv Radiol. 2007;13:68-74.

56. Yoshida D, Chen MN, Nakazawa S. Cranial metastasis of hepatocellular carcinoma in a female. Neurol Med Chir (Tokyo). 1993;33:839-44

Original recebido em junho de 2009

Aceito para publicação em julho de 2009

\section{Endereço para correspondência}

Carlos Umberto Pereira

Avenida Augusto Maynard, 245/404

49015-380 - Aracaju, SE

E-mail: umberto@infonet.com.br 